\title{
Prediction of Air Pressure Change Inside the Chamber of an Oscillating Water Column-Wave Energy Converter Using Machine-Learning in Big Data Platform
}

\author{
Dongwoo Seo, Taesang Huh *(D, Myungil Kim, Jaesoon Hwang and Daeyong Jung
}

check for updates

Citation: Seo, D.; Huh, T.; Kim, M.; Hwang, J.; Jung, D. Prediction of Air Pressure Change Inside the Chamber of an Oscillating Water

Column-Wave Energy Converter Using Machine-Learning in Big Data Platform. Energies 2021, 14, 2982.

https://doi.org/10.3390/en14112982

Academic Editors: Eugen Rusu and Mohamed Benbouzid

Received: 31 March 2021

Accepted: 18 May 2021

Published: 21 May 2021

Publisher's Note: MDPI stays neutral with regard to jurisdictional claims in published maps and institutional affiliations.

Copyright: (c) 2021 by the authors. Licensee MDPI, Basel, Switzerland. This article is an open access article distributed under the terms and conditions of the Creative Commons Attribution (CC BY) license (https:/ / creativecommons.org/licenses/by/ $4.0 /)$.
Korea Institute of Science and Technology Information (KISTI), Yuseong-gu, Daejeon 34141, Korea; seodongwoo@kisti.re.kr (D.S.); mikim@kisti.re.kr (M.K.); jshwang@kisti.re.kr (J.H.); daeyongjung@kisti.re.kr (D.J.) * Correspondence: tshuh@kisti.re.kr

\begin{abstract}
Wave power is an eco-friendly power generation method. Owing to the highly volatile nature of wave energy, the application of prediction techniques for power generation, failure diagnosis, and operational efficiency plays a key role in the successful operation of wave power plants (WPPs). To this end, we propose the following approaches: (i) deriving the correlation between highly volatile data such as wave height data and sensor data in an oscillating water column (OWC) chamber; (ii) development of an optimal training model capable of accurate prediction of the state of the wave energy converter (WEC) based on the collected sensor data. In this study, we developed a big data analysis system that can utilize the machine learning framework in KNIME (an open analysis platform), and to enable smart operation, we designed a training model using a digital twin of an OWC-WEC that is currently in operation. Using various machine learning models, the pressure of the OWC chamber was predicted, and the results obtained were tested and evaluated to confirm its validity. Furthermore, the prediction performance was comparatively analyzed, demonstrating the excellent performance of the proposed CNN-LSTM-based prediction model.
\end{abstract}

Keywords: oscillating water column; wave energy converter; machine-learning; pressure prediction model; big data platform; HPC cloud

\section{Introduction}

In recent years, the problems of global warming, environmental pollution, and depletion of natural resources caused by the use of fossil fuel-based energy, and the safety problem of nuclear energy have triggered the need for an alternative and permanent energy source, drawing attention toward renewable energy sources such as ocean energy, solar energy, and wind power. Against this backdrop, the South Korean government, in line with its incentives and planned electric power target, has announced its long-term plan to increase the proportion of renewable energy, which is currently $6 \%$ of the total power generation portfolio, to at least $20 \%$ by 2030 and $30 \%$ by 2040 [1]. Ocean energy, an attractive renewable energy source, generates electricity by utilizing tidal power, wave power, ocean current, and temperature differences of the ocean. Among these methods of power generation, wave energy generation is an environmentally friendly method of obtaining energy from the ocean, which accounts for 71\% of the earth's surface. Once a wave power plant (WPP) is installed, it can serve as a revolutionary energy source for countries by the coast, such as South Korea, owing to its low maintenance and operation costs [2]. Structures for wave energy converters (WECs) can be classified into three categories according to the conversion method of kinetic energy from ocean waves: (i) overtopping devices, (ii) wave-activated bodies, and (iii) oscillating water columns (OWC). Among these three structures, OWCs are designed to generate a reciprocating air flow through the turbines, thereby driving generators to produce energy. Although its conversion efficiency is low, the structure of the power generation system is separated from seawater, resulting in high reliability and 
safety as well as advantages in terms of maintenance, which have been reported in the related literature [3-5]. Globally, some representative OWC plants include the KVAENER plant in Norway [6], LIMPET plant in Scotland [7,8], Pico plant in Portugal [9,10], Mutriku plant in Spain [11], and Yongsu plant in South Korea. In South Korea, the West Sea around Jeju Island, in particular, has been reported for its high wave energy, facilitating the use of a WEC [12]. This is because, in the winter season, the northwest seasonal winds dominate under the specific pressure patterns such as the west-high-east-low pattern due to the development of Siberian high pressure, and the summer season is characterized by southeast or southwest seasonal winds. In winter, the wave energy is high due to the influence of the continental climate, with the highest average wave energy density being observed in December. During the typhoon season of August and September, the variation and the peak-to-average ratio of wave energy were the highest [13]. The construction of the Yongsu WPP, $1.2 \mathrm{~km}$ away from Jeju Island, was completed in July 2016 as a fixed-type OWC-WEC and has been operational since then. In general, the electric power extracted from the wave energy of an OWC-WEC is in the range of 60-500 kW, and the Yongsu WPP is currently undergoing tests with a target power generation of $500 \mathrm{~kW}$ [14]. The wave energy is converted into air flow generated by the oscillation of the water level inside the OWC air chamber, driving the air turbines and recovering electrical energy. The energy efficiency is maximized by resonance when the frequency of the incoming waves is equal to the natural frequency of the air chamber. The pressure prediction of the OWC chamber is not only the most direct parameter for improving the energy efficiency, but is also a necessary technology to prevent failure at instantaneous high pressure, which is a major issue in the operation of the Yongsu WPP generator.

The purpose of this study is to predict the pressure inside the OWC chamber, which will enable the control of the generator turbines, thereby maximizing electric power generation and preventing failure from instantaneous peak pressure. Based on the sensor data collected from the OWC-WEC, we designed a prediction model applying data preprocessing, including data correlation and machine learning, and verified the validity of the pressure prediction results in the OWC chamber. This paper is organized as follows: Section 2 outlines the related works that present an overview of OWC-WEC, and the research trend of machine learning used in OWC-WEC. Section 3 outlines the big data platform based on the HPC environment for artificial intelligence (AI) analysis of OWC-WEC datasets and the design of the OWC pressure prediction model. The datasets were defined, and significant features were derived from parameters with high correlation through correlation analysis. The section discusses a method of constructing an input dataset suitable for machine learning using data ingestion, correlation analysis of input data, data preprocessing, and noise removal. The prediction model and validation are detailed in this section. Section 4 highlights the superiority of the proposed model by comparing its performance with those of existing analysis models. Finally, Section 5 presents the conclusion and expected implications of the findings of this study and future research plans.

\section{Background}

\subsection{OWC-WEC}

An OWC-WEC converts wave energy into mechanical energy and subsequently converts mechanical energy into electrical energy. Figure 1 presents an overview of Yongsu OWC-WEC: (a) the study site of the OWC-WEC located $1.2 \mathrm{~km}$ offshore in front of Yongsuri in the region of Jeju Island, (b) schematics of the internal structure of the OWC-WEC, (c) arrangement for WPP operation, and (d) position of sensors in the turbine. The main characteristics of Yongsu OWC-WEC are presented in Table 1. The up-down motion of the air inside the OWC chamber is due to waves, leading to the reciprocating flow of air between the inside and outside of the chamber. 


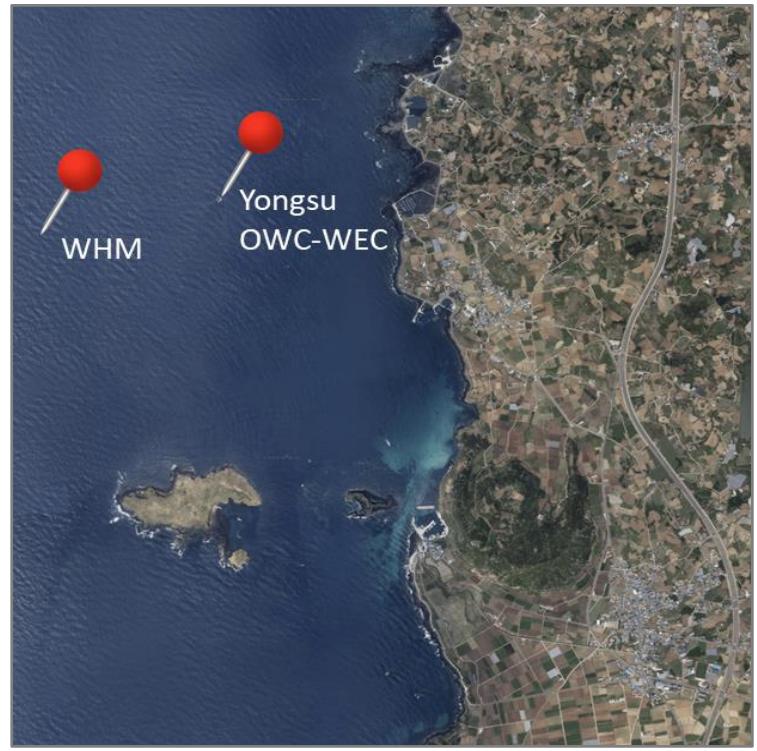

(a)

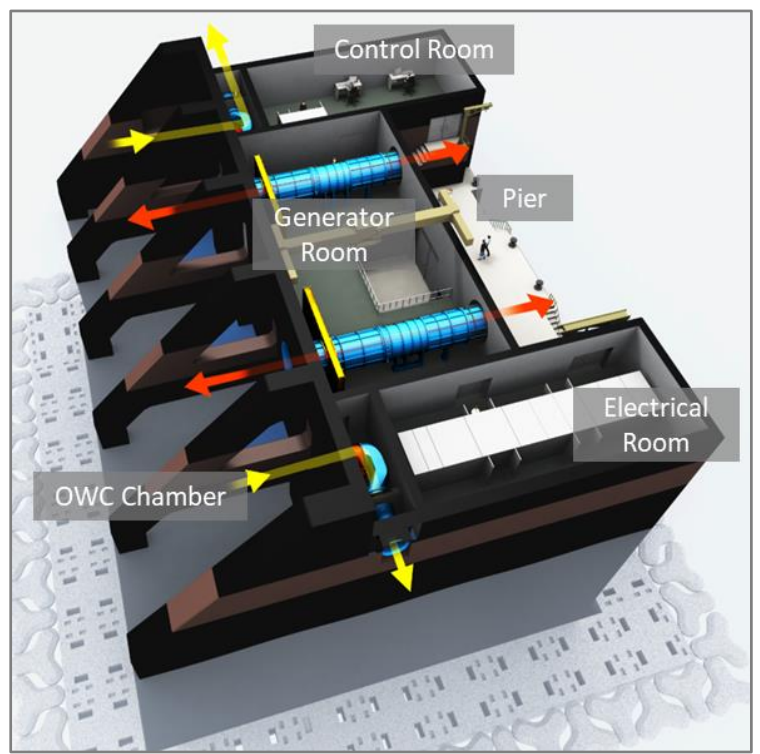

(c)

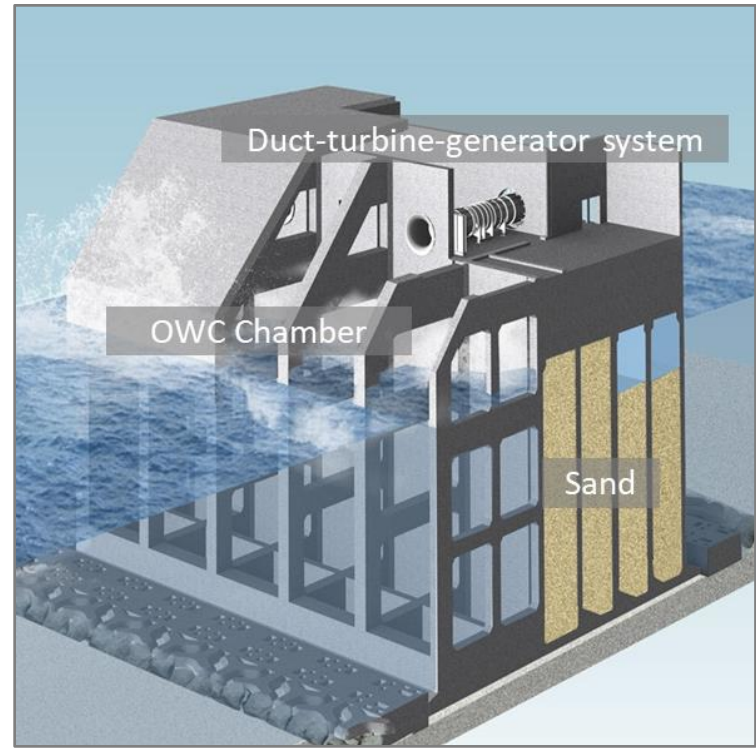

(b)

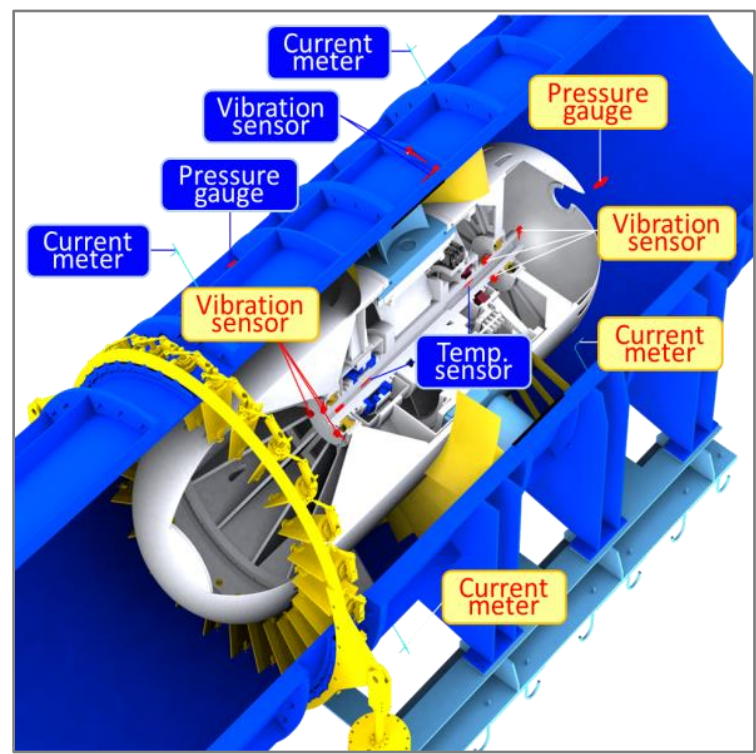

(d)

Figure 1. Yongsu OWC-WEC: (a) Location map; (b) Construction diagram; (c) Arrangement for operation; (d) Sensors in the turbine.

Table 1. Main characteristics of Yongsu OWC-WEC.

\begin{tabular}{cc}
\hline Characteristics & Description \\
\hline Capacity & $500 \mathrm{~kW}(250 \mathrm{~kW} \times 2)$ \\
External Dimensions & $37.2 \mathrm{~m}(\mathrm{~L}) \times 31.2 \mathrm{~m}(\mathrm{~B}) \times 27.5 \mathrm{~m}(\mathrm{H})$ \\
Weight & $10,500 \mathrm{ton}$ \\
Turbine & Impulse turbine of $1.8 \mathrm{~m}($ dla. $), 26$ blades \\
Generator & Synchronous generator(SG), Dual Fed Induction generator(DFIG) \\
\hline
\end{tabular}

The reciprocating air flow passes through the blade and rotates the turbine, thereby converting the wave energy into mechanical energy. The mechanical energy transferred to the turbine is then converted into electrical energy through the WEC. However, owing to the characteristics of renewable energy, the generated voltage is not constant, and the 
transferred energy is converted to electrical energy with a constant voltage and frequency through a power conversion system (PCS). That is, because the output of the WEC is electrical energy with varying voltage and frequency, the PCS is essential for operating the WEC in connection with power grids $[15,16]$.

\subsection{Machine-Learning (ML) in OWC-WEC}

ML is a subset of AI; it uses an algorithm in which data are analyzed and learned, and decisions and predictions are made on the output of new input data. ML can be categorized into supervised and unsupervised learning according to the learning type. Supervised learning uses labeled data that includes both input and output data for learning. Using the input and output of the training data, the function of the applicable system is inferred, and when there is a new input, the function is used to predict the corresponding output. Supervised learning can be categorized into regression, which is a prediction for continuous output data, and classification, which is a prediction for discontinuous output data. Unsupervised learning uses unlabeled data with only input data, and clustering is typically used in learning in which data with similar features are grouped using the features of the input data. For the pressure prediction in this study, a regression analysis algorithm that infers continuous output data for continuous input data was considered suitable. Representative regression analysis algorithms include linear regression (LR), support vector regression (SVR), decision regression tree (DT), random forest regression (RF), multilayer perception (MLP), and deep learning (DL). Algorithms frequently used in DL include convolutional neural networks (CNNs), recurrent neural networks (RNNs), and long shortterm memory (LSTM) [17-19]. With the aim of achieving the operational efficiency of WPPs, several studies have developed prediction models for wave energy, OWC chamber pressure, and electric power. There are three categories of methodologies: statisticsbased modeling, machine learning-based modeling, and DL-based modeling, which are described in Table 2. In the statistical model category, wave energy is predicted by applying an autoregressive model [20]. The results showed that the temporal resolution of the observed data affected the performance of the prediction model. In addition, wave energy is predicted by applying a LR [21]. This method mainly uses general LR with a simple design and removes unnecessary parameters to improve the stability of the predictive performance. However, this method has a disadvantage in that it cannot predict the irregularity of the time-series. In the machine-learning-based modeling category, neural networks were used to predict the wave energy of the WPP and the pressure in the chamber [22-24]. They acquired data from a real WPP during operation and performed short-term predictions to increase operational efficiency. In addition, by applying autoregressive modeling and NNs and performing predictions considering the characteristics of the time series, the performance stability improved. However, in the existing machine-learning method, severe overfitting occurs when the variation of the time-series increases or the amount of data increases, and overfitting makes accurate prediction difficult. Recently, predictive studies of the wave energy and electrical energy of WPPs have been conducted using DL to address some of the existing limitations. Using data from an actual WPP in operation and sensors, higher performance was achieved than the existing prediction method. The DL method was used to reduce randomness and noise from sensor data and extract features suitable for prediction. This method can automatically extract and model key features, even in cases of large amounts of data and data with complex attributes [25,26]. However, there has been no prior research investigating the prediction of temporal information in time-series data of the pressure in the chamber and the spatial correlation between parameters. In most studies, general sections were selected from time-series data, and temporal information was modeled for prediction, and prediction methods without consideration of temporal information were used. Therefore, in an environment highly subject to volatile energy, such as a WPP, a modeling and learning method that takes into account irregular temporal information and spatial information of parameters is required. 
Table 2. Deep-learning models in WEC.

\begin{tabular}{|c|c|c|c|}
\hline Category & Author & Method & Short Description \\
\hline \multirow[t]{2}{*}{ Statistical based modeling } & Fusco, F.; Ringwood, J.V. [25] & LR, DT & $\begin{array}{l}\text { Presents an approach where the wave } \\
\text { elevation is treated as a time series and it is } \\
\text { predicted only from its past history }\end{array}$ \\
\hline & Simonetti. I. [26] & LR, DT & Presents an optimization of OWC WEC \\
\hline \multirow{3}{*}{$\begin{array}{c}\text { Machine } \\
\text { learning- based modeling }\end{array}$} & $\begin{array}{l}\text { Wanan Sheng, } \\
\text { Tony Lewis. [27] }\end{array}$ & MLP & $\begin{array}{l}\text { Presents a short-term prediction of the } \\
\text { random process by an ANN, which can } \\
\text { provide future information for the } \\
\text { control system }\end{array}$ \\
\hline & $\begin{array}{l}\text { Paparella, F.; Monk, K.; } \\
\text { Winands, V. [28] }\end{array}$ & MLP & $\begin{array}{l}\text { Presents a prediction of the wave elevation } \\
\text { inside the chamber of the OWC for the Pico } \\
\text { OWC plant in the Azores }\end{array}$ \\
\hline & $\begin{array}{l}\text { Monk, K.; Conley, D.; } \\
\text { Winands, V. [29] }\end{array}$ & MLP & $\begin{array}{c}\text { Presents a short-term wave forecasting using } \\
\text { an autoregressive model }\end{array}$ \\
\hline \multirow[b]{2}{*}{$\begin{array}{c}\text { Deep } \\
\text { learning- Based Modeling }\end{array}$} & Ni, C.; Ma, X. [30] & $\mathrm{CNN}$ & \multirow{2}{*}{$\begin{array}{l}\text { Presents a prediction of WEC using a } \\
\text { convolutional NN with Multiple Inputs } \\
\text { Design a novel custom power demand } \\
\text { forecasting algorithm based on the LSTM } \\
\text { deep-learning method regarding the recent } \\
\text { power demand patterns }\end{array}$} \\
\hline & $\begin{array}{l}\text { Choi, E.; Cho, S.; } \\
\text { Kim, D.K. [31] }\end{array}$ & LSTM & \\
\hline
\end{tabular}

\section{Materials and Methods}

In this study, a platform for large-scale sensor data ingestion and support of timeconsuming AI calculations was developed. The structure of the high-performance data analysis and processing platform based on big data is illustrated in Figure 2 and each key element can be described as follows:
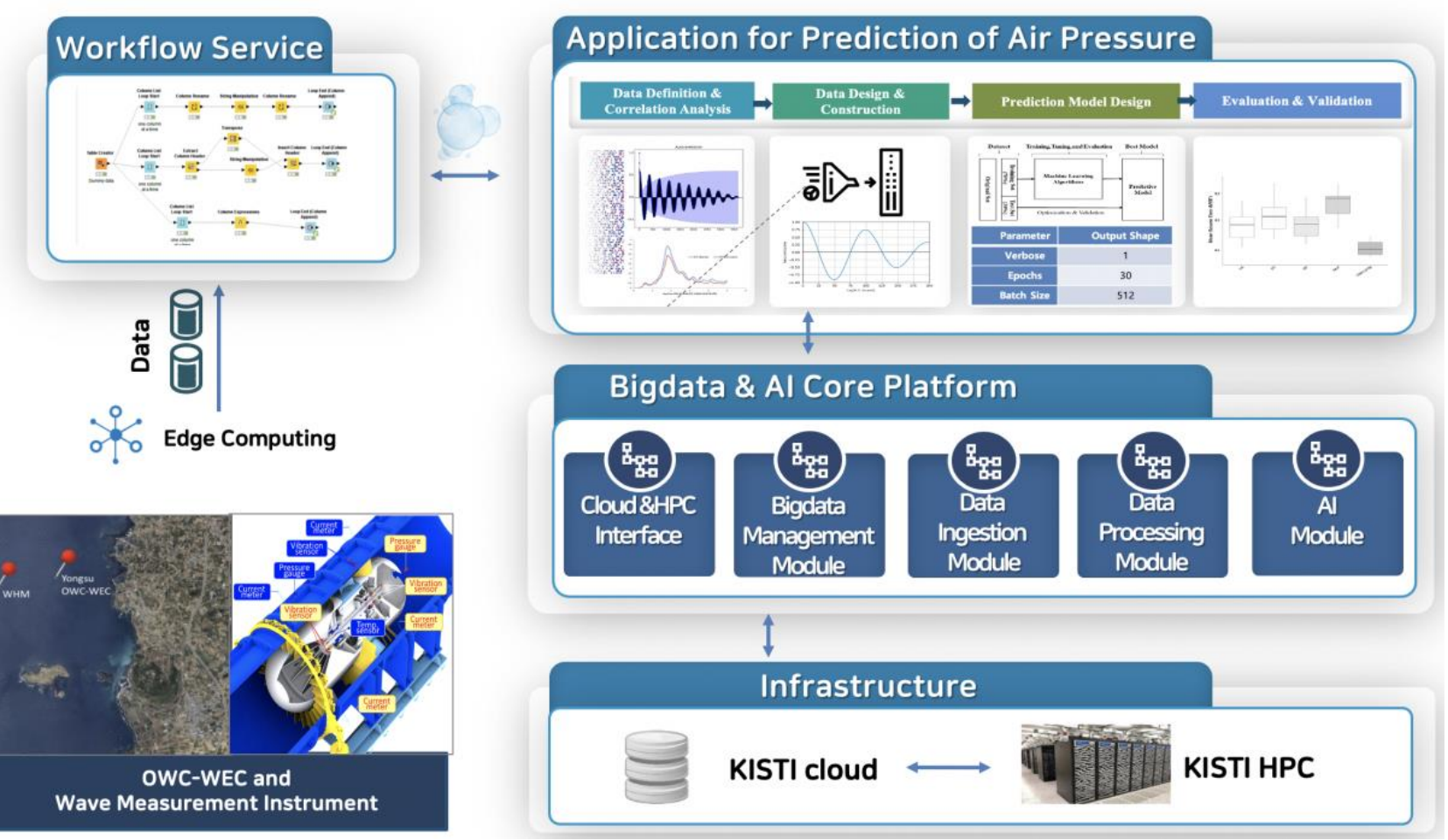

Figure 2. Structure of proposed big data and AI platform. 
1. Workflow Service: Supports blend tools from WEC domains with big data and AI core platform in a single workflow, including scripting in R and Python and ML

2. Application: Provides functions such as correlation analysis, real-time data monitoring, data browsing, and result analysis, and monitoring.

3. Big data and AI Core Platform: Supports cloud and HPC user interface, big data management module, data ingestion module, and AI module.

4. Infrastructure: Provide KISTI cloud and KISTI HPC resource

To develop a training model based on real data, external wave data of the OWC wave power generation system and sensor data generated from the OWC wave height meter (WHM) were acquired. Data preprocessing, such as filtering, was performed with the acquired raw data, and the datasets were classified into training sets and test sets, which were used to develop the training model. This section describes the entire process of developing a pressure prediction model. The pressure prediction model was developed using the proposed big data and AI platform, to support real-time sensor big data ingestion and analysis. Figure 3 shows a schematic of the overall process for the development of a pressure prediction model.

\section{Overall Process for Prediction of Air Pressure}

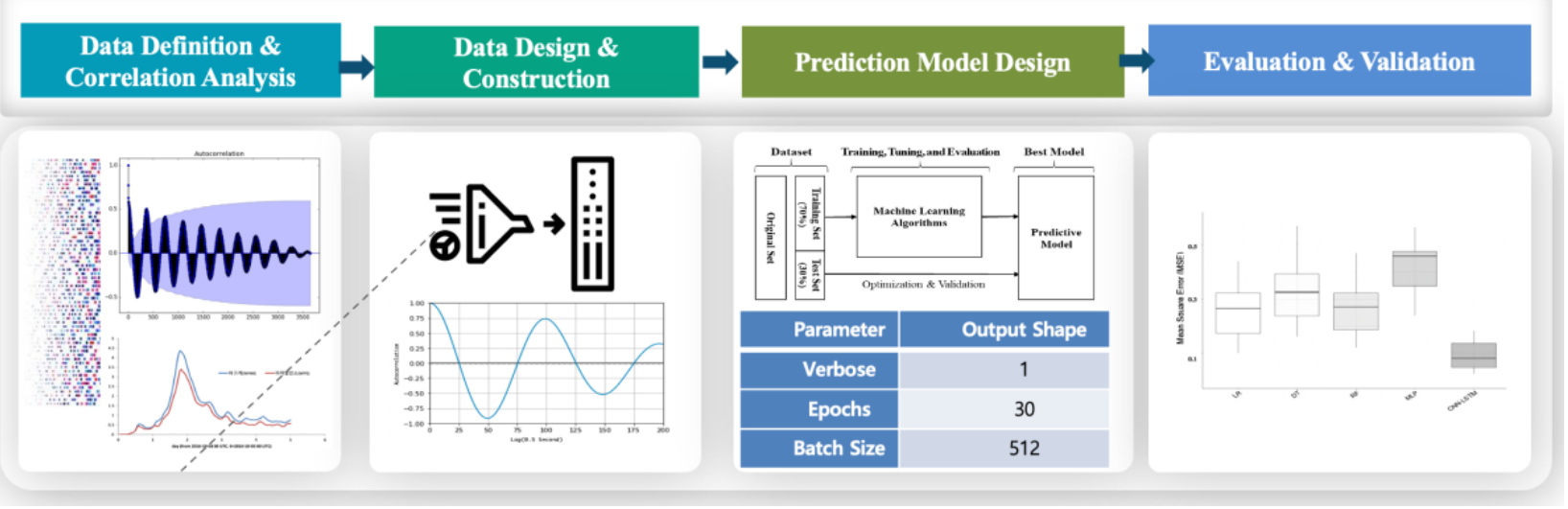

Figure 3. Process of the pressure prediction model based on machine-learning in OWC-WEC.

\subsection{Data Definition and Correlation Analysis}

For data ingestion, WHM and OWC-WPP data were selected as follows: (i) WHM data (e.g., wave height, wave period, wave direction) and (ii) OWC-WPP data (e.g., flow, pressure, and power). To examine the effect of WHM data, we performed a correlation analysis between the wave height data measured from WHM for $48 \mathrm{~h}$ (see Table 3, Figure 4) and OWC-WPP data (see Table 4, Figure 5).

Table 3. WHM attribute table.

\begin{tabular}{cc}
\hline Attribute & Description \\
\hline $\mathrm{T}$ & Mean wave period \\
$\mathrm{H}$ & Significant wave height \\
Direction & Mean wave direction \\
\hline Collecting cycle: 30 min., Collecting duration: $48 \mathrm{~h}$ \\
\hline
\end{tabular}




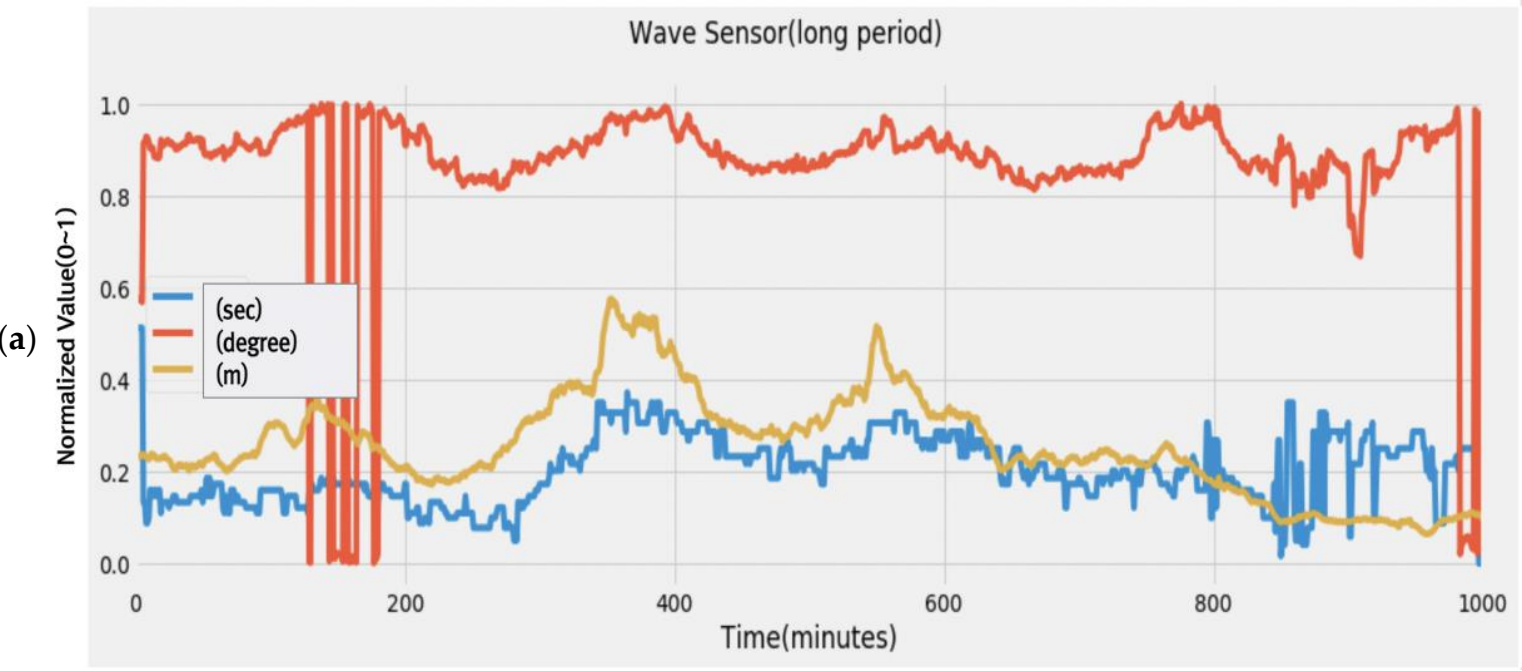

Wave Sensor(short period)

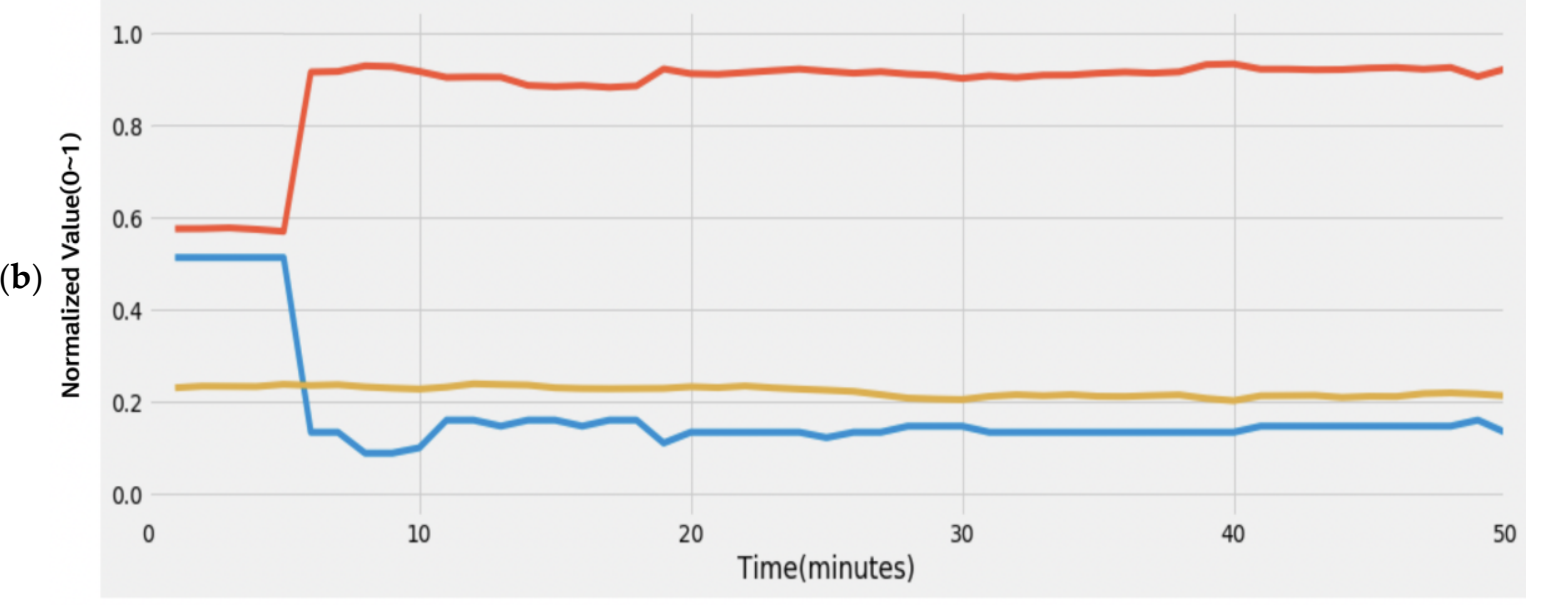

Figure 4. Raw data obtained in the WHM: (a) Long period; (b) Short period.

Table 4. OWC sensor attribute table.

\begin{tabular}{cc}
\hline Attribute & Description \\
\hline Flow & Flow velocity \\
Press & Chamber pressure \\
Torque & Motor torque \\
RPM & Rotations per minute \\
Power & electrical energy \\
& Ingestion cycle $(1 \mathrm{~s})$, Ingestion time $(48 \mathrm{~h})$ \\
\hline
\end{tabular}

Figure 6 shows the workflow for data preprocessing and correlation analysis. To preprocess data for correlation analysis, data normalization was performed. The simulated waves nearshore (SWAN) model [27] and statistical linear correlation [28] were used for the analysis. The wave energy and pressure data were based on 30 min intervals, considering the minimum interval of the measured wave energy. Figure 7 shows the results of the analysis with 30 min intervals over the course of $48 \mathrm{~h}$. The SWAN model was used to analyze the impact of wave height sensors and WPPs, which are separated in the distance. The results showed that a time delay and deviation occurred because of the distance of $1 \mathrm{~km}$ between the Yongsu WPP and WHM, making it difficult to utilize the wave height data for the target WPP in this study. 
(a)
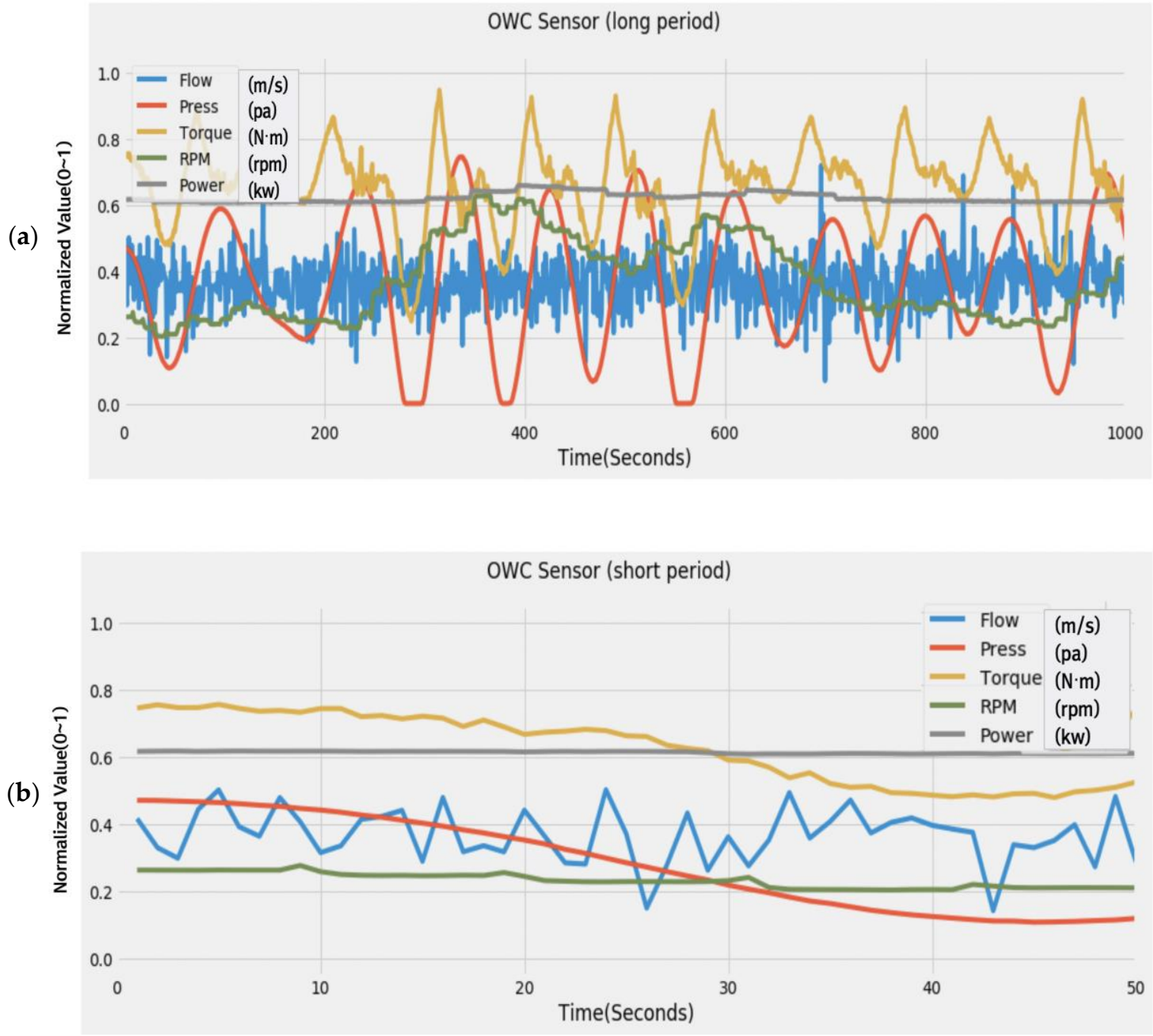

Figure 5. Raw data obtained in the OWC: (a) Long period; (b) Short period.

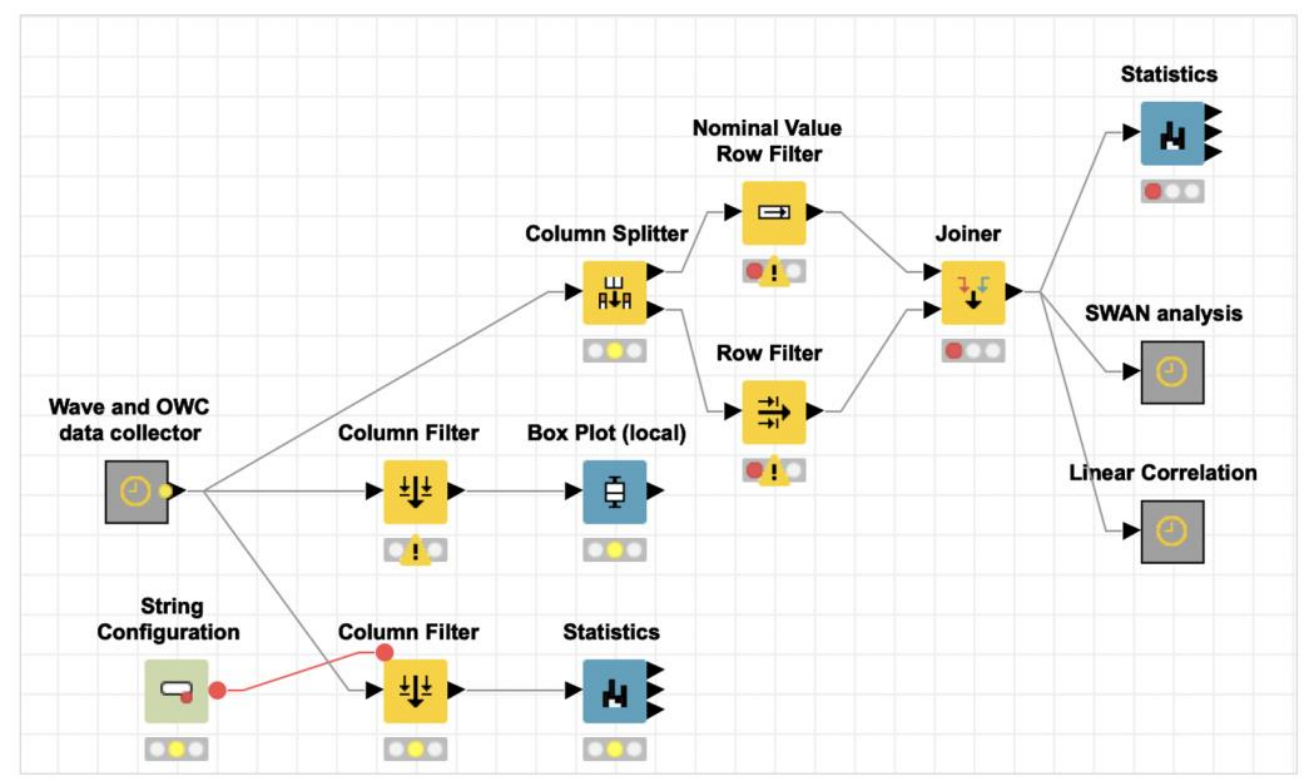

Figure 6. Correlation analysis. 


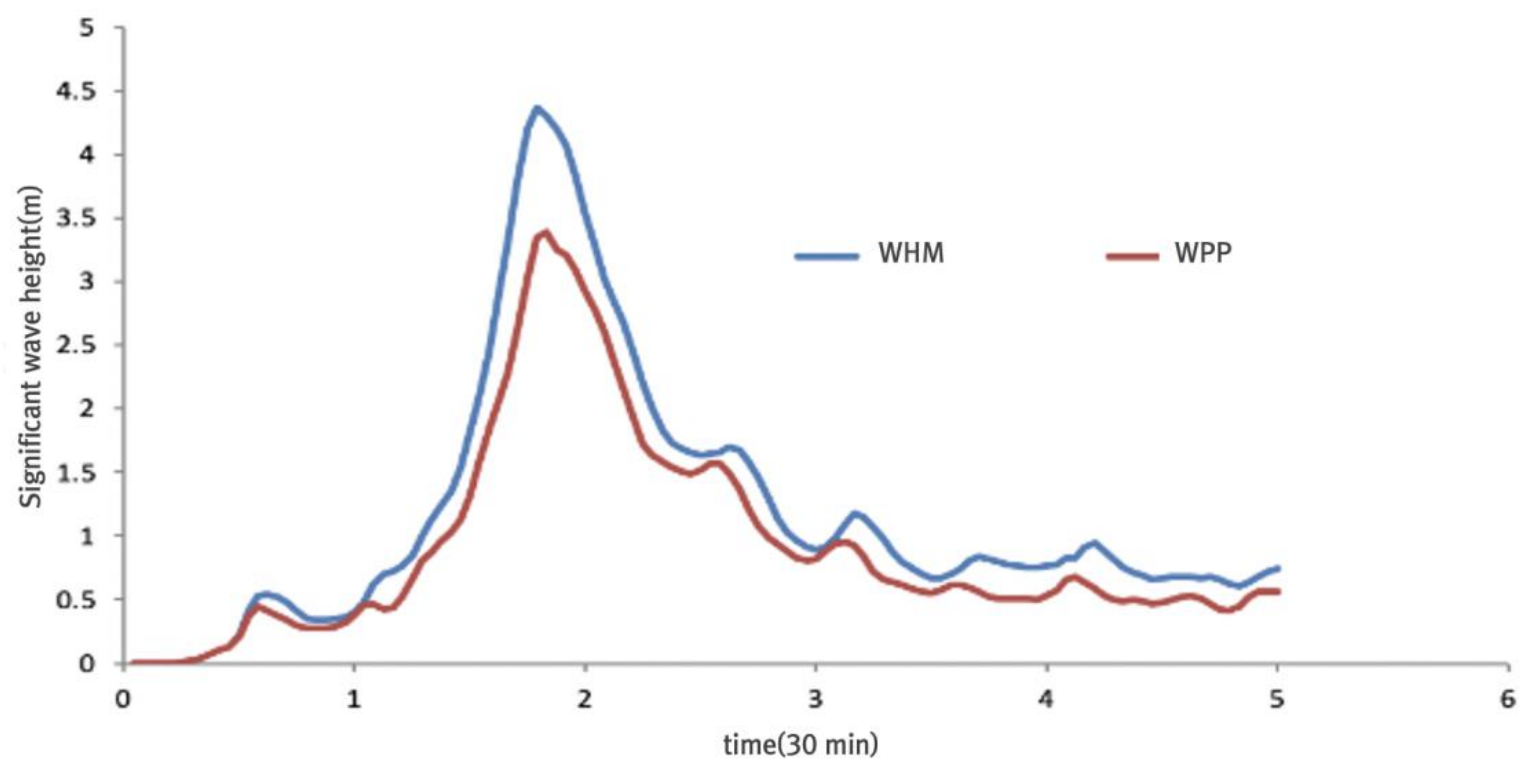

Figure 7. Analysis with SWAN.

The utilization of wave height data is limited owing to the time-delay problem, considering the $30 \mathrm{~min}$ intervals of the measured wave height. The reason for using OWC and WEC data is that, as shown in Figure 7, OWC generates pressure energy from the actions of wave energy, and the pressure is converted into energy such as flow velocity to operate the turbines; thus, it is judged that there is a close correlation between the two sets of data.

In order to analyze the pressure change due to air flow in both directions in the OWC chamber, the data samples were collected every second; the minimum interval between OWC data and the wave height data with measured electrical power. Table 5 shows the result of correlation analysis between OWC data and WEC data acquired in $1 \mathrm{~s}$ intervals for $48 \mathrm{~h}$. From the analysis, there is a high positive correlation between the pressure data of OWC and WEC data, such as electrical energy. Therefore, it can be assumed that the OWC chamber pressure is one of the key parameters for the operation of WEC; thus, data prediction using machine learning can be considered instrumental in the operation of OWC-WEC.

Table 5. Result of correlation analysis.

\begin{tabular}{|c|c|c|c|c|c|}
\hline & $\begin{array}{c}\text { Press } \\
\text { (Chamber } \\
\text { Pressure) }\end{array}$ & $\begin{array}{c}\text { Flow } \\
\text { (Fluid Velocity) }\end{array}$ & $\begin{array}{c}\text { Torque } \\
\text { (Motor Torque) }\end{array}$ & RPM & Power \\
\hline $\begin{array}{c}\text { Press } \\
\text { (chamber } \\
\text { pressure) }\end{array}$ & 1 & 0.976678 & 0.919996 & 0.913444 & 0.905568 \\
\hline $\begin{array}{c}\text { Flow } \\
\text { (fluid velocity) }\end{array}$ & 0.314410 & 1 & 0.452299 & 0.452299 & 0.452299 \\
\hline $\begin{array}{c}\text { Torque } \\
\text { (motor torque) }\end{array}$ & 0.392105 & 0.681115 & 1 & 0.952299 & 0.952299 \\
\hline RPM & 0.324449 & 0.612237 & 0.892699 & 1 & 0.952299 \\
\hline Power & 0.352299 & 0.652211 & 0.852299 & 0.852299 & 1 \\
\hline
\end{tabular}

\subsection{Dataset Design and Construction}

\subsubsection{Data Preprocessing}

To prepare the input data, features are processed and generated from the given raw data. Data preprocessing requires the creation of features from domain knowledge, which 
refers to creating or selecting a column (feature) of the data table for ML. This type of feature engineering has a large impact on the model performance, and the preprocessing techniques outlined in Table 6 are used to generate the training data. Because the prediction model generally operates on the assumption of using correct input data, a data cleansing method for generating training data is derived through the analysis of WHM data, internal pressure data, and power generation data. First, in order to perform a correlation analysis of features, processing to match the interval between sensor data with different measurement periods and preprocessing to match the format were performed. A dataset building plan was established in which the target feature (pressure) was selected, and based on the target feature, correlation analysis by sensor data feature was performed to select the input feature.

Table 6. Feature engineering for data preparation.

\begin{tabular}{|c|c|}
\hline Item & Description \\
\hline Removal of columns with many missing values & $\begin{array}{l}\text { - Columns with many missing values } \\
\text { cannot be used as input data }\end{array}$ \\
\hline Handling missing values & $\begin{array}{l}\text { - Replace with a mean value or a } \\
\text { calculated value } \\
\text { - In general, missing values are substituted } \\
\text { with } 1 \text { or } 0 .\end{array}$ \\
\hline Integer Encoding & $\begin{array}{l}\text { - When there is text-type data } \\
\text { - After classifying by word, the data is } \\
\text { converted to index }\end{array}$ \\
\hline $\begin{array}{l}\text { Removal of redundant and highly } \\
\text { correlated variables }\end{array}$ & $\begin{array}{l}\text { - Minimizing duplicate data, redundant } \\
\text { data, or highly correlated variables } \\
\text { - Application of scatter plot and principal } \\
\text { component analysis }\end{array}$ \\
\hline Modification of biased values & $\begin{array}{l}\text { - Continuous variables should follow a } \\
\text { normal distribution } \\
\text { - After reviewing the distribution trend, } \\
\text { the biased values are modified } \\
\text { - } \quad \text { Review: Use of statistical scale and } \\
\text { histogram } \\
\text { - Modification/correction: use of log } \\
\text { transformation, inverse transformation, } \\
\text { square root transformation, and squares }\end{array}$ \\
\hline $\begin{array}{l}\text { Handling extreme values and } \\
\text { extraordinary values }\end{array}$ & $\begin{array}{l}\text { - } \quad \text { Use the extreme values, where possible } \\
\text { Remove outliers (values not within } \\
\pm 3 S D), \text { when they do not have } \\
\text { significant implications }\end{array}$ \\
\hline
\end{tabular}

The preprocessing workflow for correlation analysis was established as shown in Figure 8 , and the wave height data were read from the folder to obtain a file list, and input data were considered through this process. For data analysis considering time-series, nodes were created for the input and processing of the time-series and period of data, and nodes for constructing the applicable data were additionally designed to support processing such as filtering, merging, and column change of data. To resolve the problem of imbalanced data in which the proportion of data for each class is not uniform but biased, the performance of the classification algorithm is improved. The reason for identifying unbalanced classification as a problem can affect the performance of ML algorithms, so the skew may not be significant. Oversampling and undersampling are used to adjust the 
class distribution of a dataset. In particular, the data collected by the sensors of the current wave power system have unwanted noise due to the effect of wave energy, and the graph is not flattened, as shown in Figure 9a. To utilize the water level prediction data in a flow analysis model, noise needs to be removed to flatten the graph. In this study, Equation (1) was used to flatten the data by removing noise from the OWC chamber sensor data.

$$
|\mathrm{H}(j \omega)|=\frac{1}{\sqrt{1+\left(\frac{\omega}{\omega_{c}}\right)^{2 n}}} .
$$

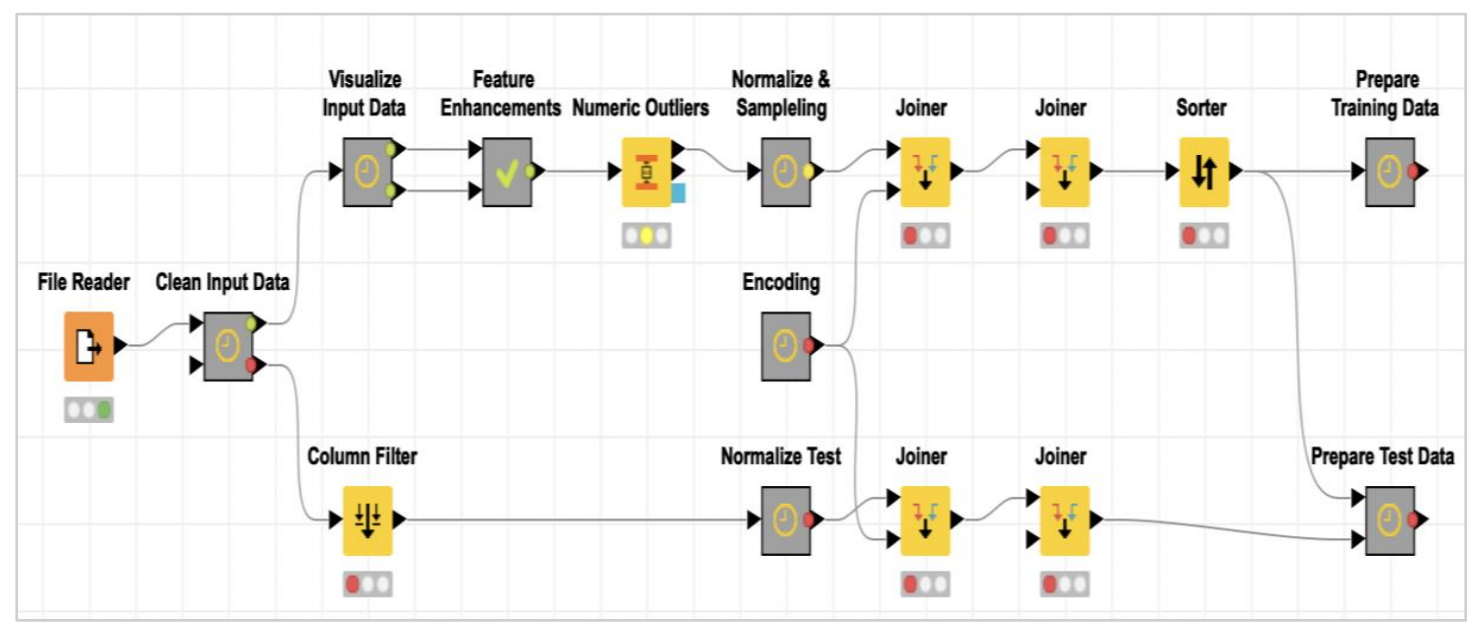

Figure 8. Preprocessing workflow to build a dataset.

(a)

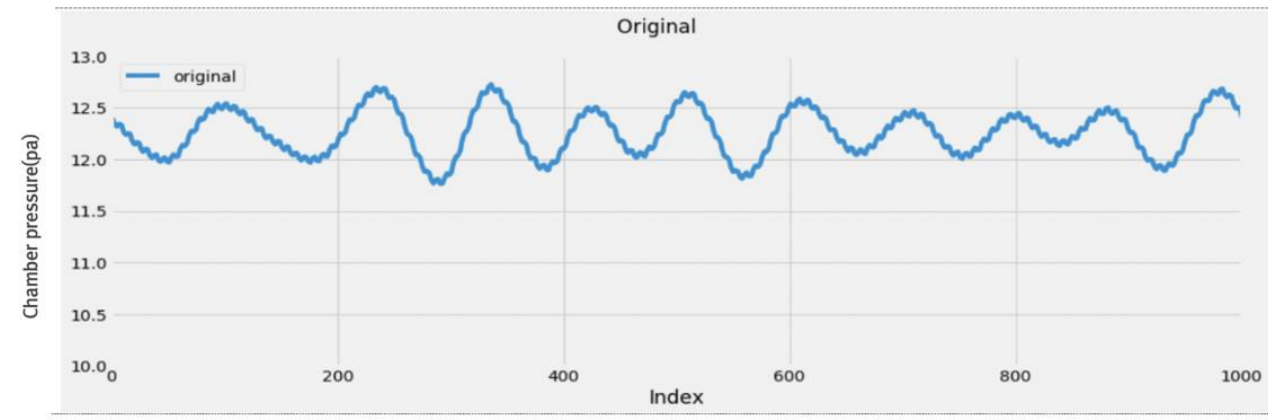

(b)

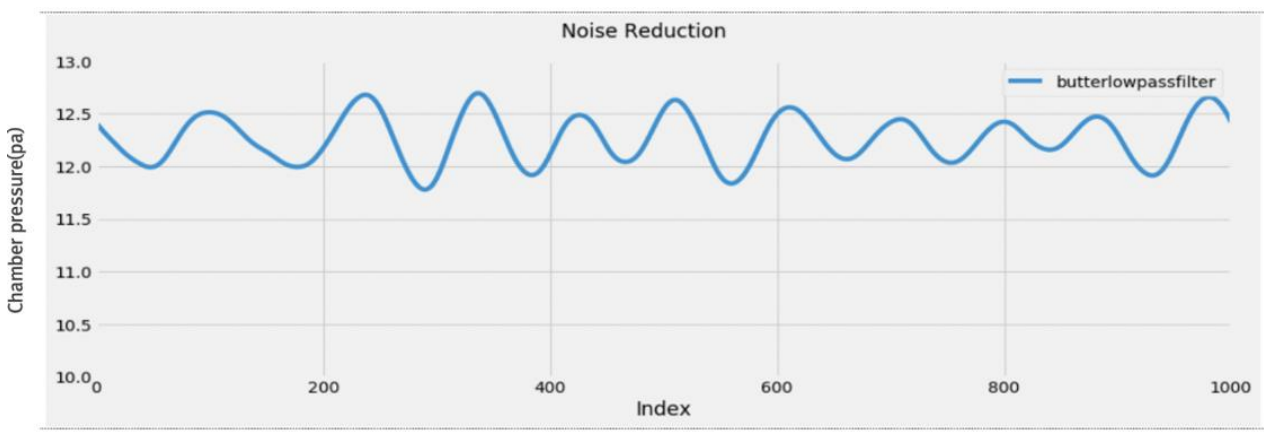

Figure 9. Air pressure signal of (a) before and (b) after noise reduction in OWC.

The Butterworth low-pass filter generates no ripples in the passband and attenuates the unwanted frequencies outside this band. The Butterworth low-pass filter selects a transfer function so that the magnitude response curve can be as flat as possible in the passband of the filter; thus, this filter is known to have a maximally flat magnitude. The 
result of flattening by applying a Butterworth low-pass filter to signals containing noise is shown in Figure 9b.

\subsubsection{Dataset Construction}

Autocorrelation analysis was performed to construct datasets reflecting the autocorrelation of the time series, as shown in Figure 10. Autocorrelation is a function representing the correlation between values taken at two time points of a random signal and helps to extract the principal components from time-series data. As a result of the autocorrelation analysis, a strong negative correlation of -0.9 at $2.5 \mathrm{~s}$ intervals and a positive correlation at 0.75 with $5 \mathrm{~s}$ intervals were discovered. Therefore, the target time for prediction was determined to be $2.5 \mathrm{~s}$, which has a high correlation, and datasets were constructed accordingly.

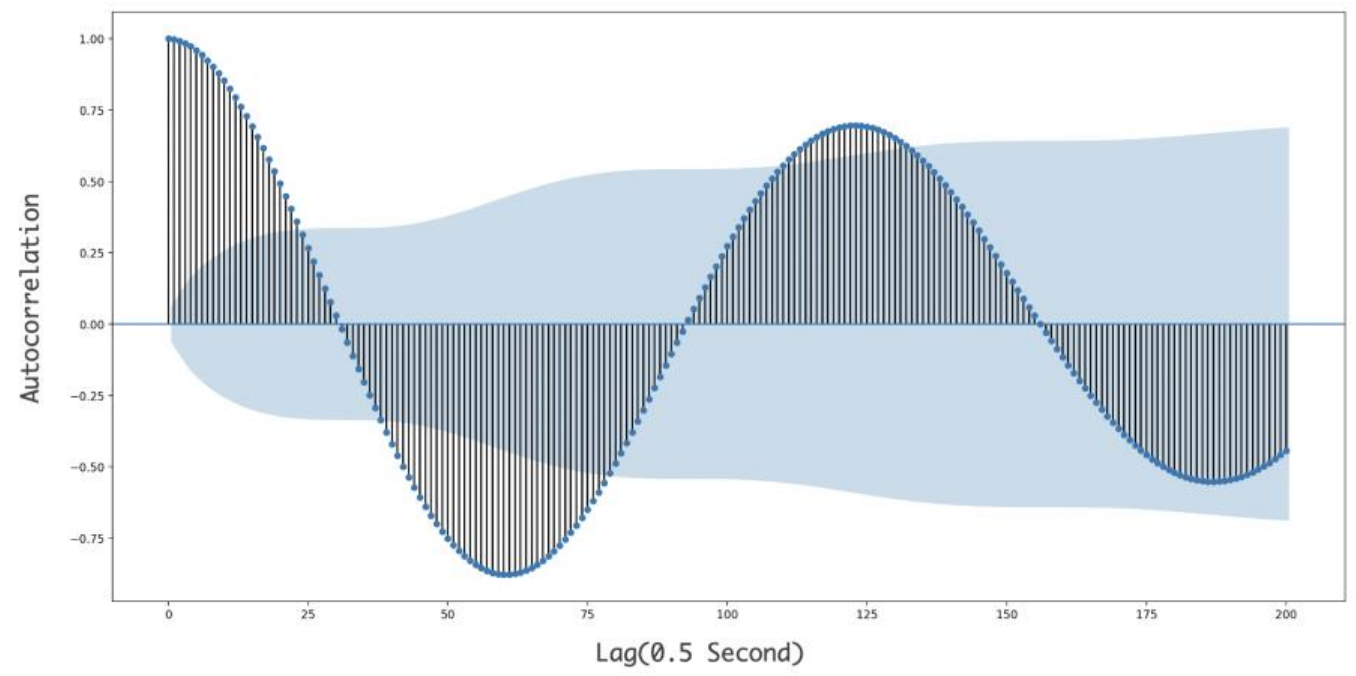

Figure 10. Result of autocorrelation analysis.

The datasets for ML can be largely classified into training sets and test sets, and if a sufficient volume of data is acquired, the datasets can be classified into training sets, test sets, and validation sets, additionally enabling validation of data. Each type of dataset can be described as follows: (i) training sets to learn data patterns from raw data; (ii) test sets to test the model performance by running the model according to an actual scenario; and (iii) validation sets for tuning and evaluation. In this prediction model, training sets for OWC pressure prediction were constructed based on the window sliding of the correlation, as shown in Figure 11.
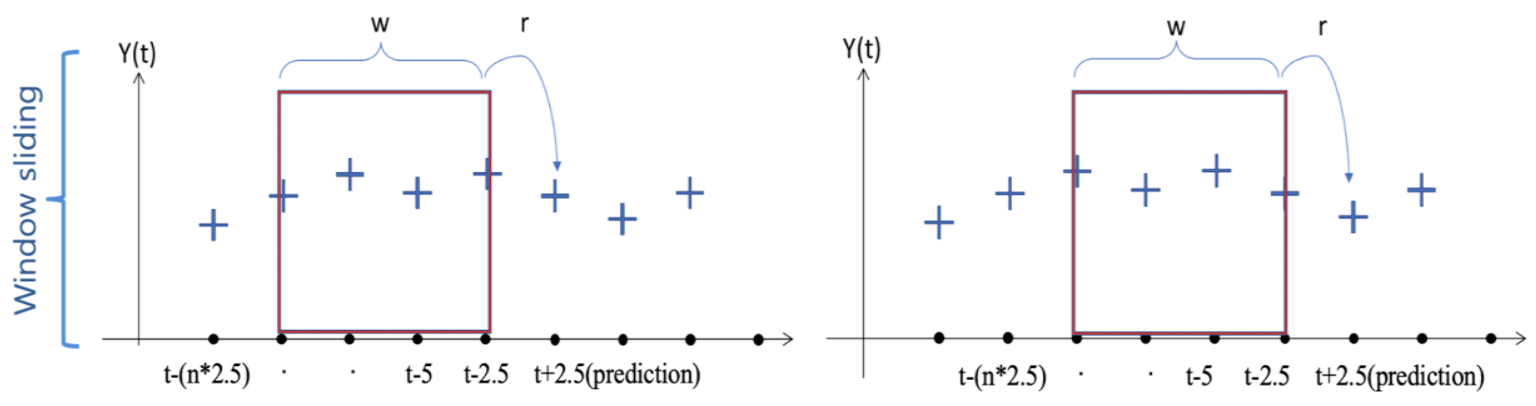

Figure 11. Target feature and prediction method. 


\subsection{Predictive Model Design}

Recently, the CNN-LSTM, an algorithm that has been used in time-series prediction has attracted attention owing to its excellent prediction performance. It extracts the spatial features of surrounding data by sliding a filter that performs a convolution operation in the convolution layer over a sequence [8]. Subsequently, LSTM, an algorithm optimized for grasping time information, is applied, and prediction is performed by incorporating temporal features. The architecture of CNN-LSTM can have various designs according to the type of layer constituting the network and tuning of parameters. CNN-LSTM consists of a convolutional layer, pooling layers, LSTM layers, and dense layers. The number of filters, kernel size, and the number of strides can be tuned for each layer. Tuning these parameters can affect the learning rate and performance according to the features of the training data. The changes in performance can be examined by varying these parameters. The identification of features of the input data plays a key role in the development of an optimal architecture for the prediction of OWC chamber water level with parameter tuning. Therefore, after predicting the OWC chamber water level through hyperparameter tuning, it was designed to select one value with the smallest error in the last fully connected layer.

Hyperparameter tuning refers to adjusting (tuning) hyperparameters to find the optimal training method by evaluating the model performance of the model with validation sets. The datasets with the highest prediction accuracy were those with all outliers removed. Table 7 lists the hyperparameters determined by hyperparameter tuning. The larger the epoch, the better the prediction result, but if it is too large, overfitting can occur leading to a significantly lower learning rate. Therefore, the size of the epoch was fixed at an optimal value of 30, and the number of units of the convolution layer, the number of layers, filter size, pooling size, and batch size were empirically determined.

Table 7. Hyperparameter.

\begin{tabular}{cc}
\hline Parameter & Output Shapes \\
\hline Verbose & 1 \\
Epochs & 30 \\
Batch Size & 512 \\
\hline
\end{tabular}

Table 8 details the overall architecture of the CNN-LSTM proposed herein. The parameters of all layers are listed, including the LSTM layer; these include the number of filters in each convolution layer, size of the convolution layer, and the number of strides.

Table 8. CNN-LSTM architecture.

\begin{tabular}{ccc}
\hline Layer (Type) & Output Shape & Param \# \\
\hline ConvLSTM2D (Activation = relu) & (None, 1, 48, 64) & 50,176 \\
Flatten & (None, 3072) & 0 \\
Repeat_Vector & (None, 1, 3072) & 0 \\
LSTM (Activation = tanh) & (None, 1, 40) & 498,080 \\
Time_Distributed (Activation = relu) & (None, 1, 40) & 1640 \\
Time_Distributed & (None, 1, 1) & 41 \\
\hline
\end{tabular}

Total params: 549,937

\subsection{Evaluation and Validation}

\subsubsection{Training Evaluation}

When training the prediction model with the training datasets, the training error was calculated using the loss function, and the weights and bias were modified to minimize the training error. Therefore, the training error is a highly useful indicator of the current state 
of the prediction model. As a general training error function, the mean squared error is used, which is given by

$$
M S E=\frac{1}{n} \sum_{i=1}^{n}\left(y_{i}-t_{i}\right)^{2} .
$$

\subsubsection{Validation}

After the completion of the training process, the previously discussed process was repeated in the same manner for the validation datasets. The error rate for a new sample is called the generalization error, and the generalization error is estimated by evaluating the prediction model with the validation sets. This estimate provides information about the performance of the predictive model for new data. Appropriately obtaining the generalization error and comparing it with the training error is crucial for performing performance comparisons. A small training error but a large generalization error indicates that the prediction model is overfitted to the training data. The total number of datasets used in this study was 700,000, which cannot be considered large. When the validation sets are too small, the model performance cannot be evaluated accurately, and the selection of the optimal model may be incorrect [7]. Cross-validation was performed to resolve this problem.

\subsubsection{Evaluation of Prediction Results (Prediction Precision)}

Root mean square error is a measure used to represent the difference between the predicted and actual values of the model. The RMSE for representing the prediction precision is given by

$$
\text { RMSE }=\sqrt{\frac{1}{n} \sum_{i=1}^{n}\left(y_{i}-t_{i}\right)^{2}} .
$$

\section{Results}

\subsection{Performance Comparison with Conventional Machine-Learning Methods}

To verify the utility of the proposed pressure prediction model based on CNN-LSTM, tests were performed using LR and a machine learning algorithm. In the performancecomparison test, 10-fold cross-validation was used, and the MSE was the lowest for the proposed CNN-LSTM among the machine-learning methods as well as LR. Figure 12 presents a box plot showing the MSE obtained using a 10-fold cross-validation test. The proposed method exhibits the best average performance. The figure shows a significant difference in MSE between the proposed CNN-LSTM and the existing methods. In addition, the results confirm that the proposed method ensures more stable performance using the distance from the mean value. Table 9 lists the parameters of the machine-learning methods used in the tests. Hyperparameters were tuned to achieve superior performance for each model to compare the CNN-LSTM with the existing methods. The hyperparameters for each model were set using the scikit-learn library, Python's representative machinelearning package, and each hyperparameter was determined empirically. $\mathrm{Xi}$ is a vector composed of $\mathrm{n}$ predictions generated from samples of energy consumption data points for all parameters, and $Y i$ is a vector composed of the observed consumption values of the predicted parameters. 


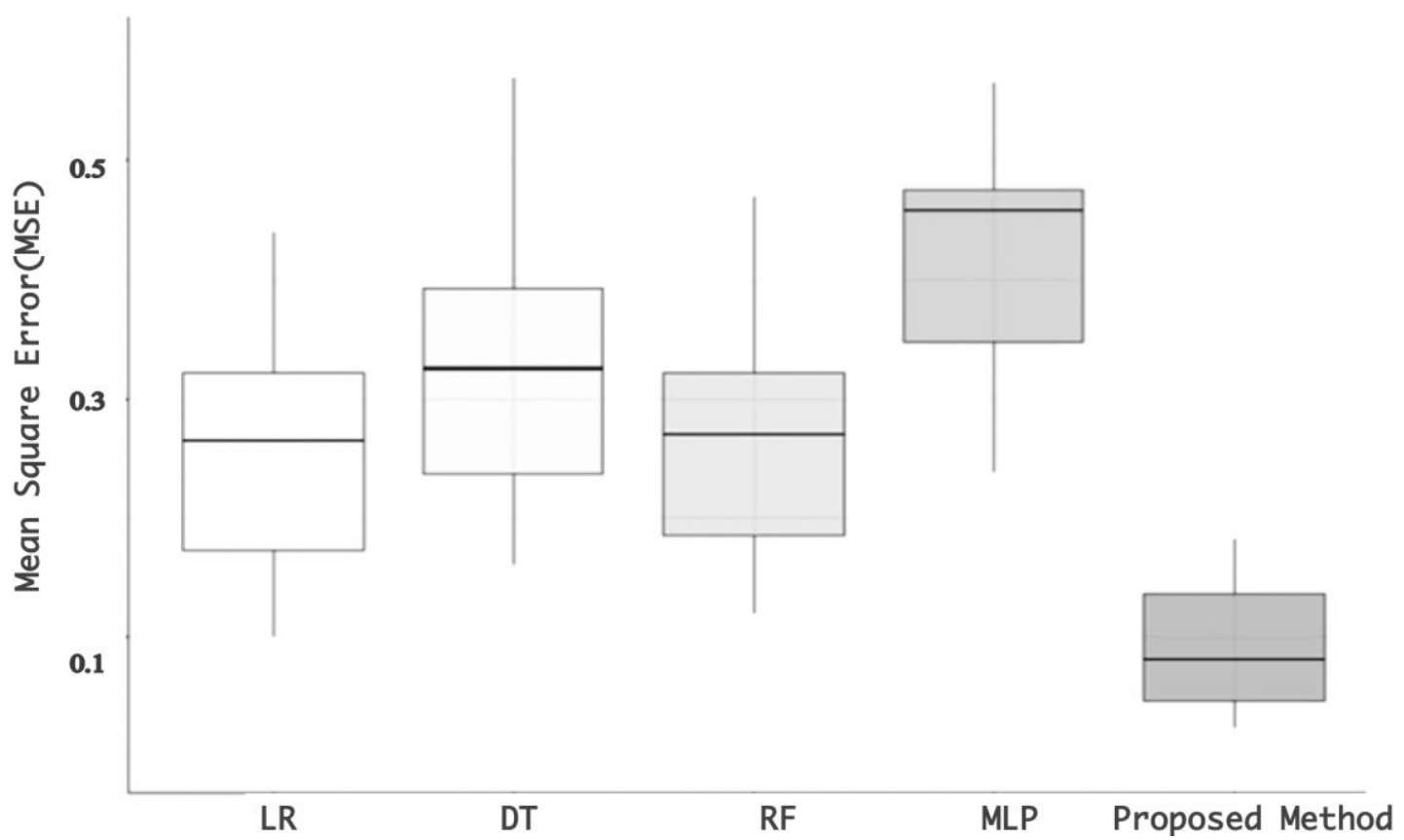

Figure 12. Accuracy of 10-fold cross-validation using machine learning.

Table 9. Parameters for machine-learning techniques.

\begin{tabular}{|c|c|c|}
\hline No. & Model & Description \\
\hline 1 & Linear Regression (LR) & Fit_intercept $=$ True, normalize $=$ False, $\mathrm{n} \_$jobs $=$None \\
\hline 2 & Decision Tree Regression (DT) & $\begin{array}{l}\text { Splitter }=\text { "best", maximum depth = None, } \\
\text { min samples split }=2 \text {, min sample leaf }=1\end{array}$ \\
\hline 3 & Random Forest Regression (RF) & $\begin{array}{l}\text { No of estimators }=10, \text { maximum depth }=\text { None, } \\
\text { min samples split }=2, \text { min sample leaf }=1\end{array}$ \\
\hline 4 & Multilayer perceptron (MLP) & $\begin{array}{c}\text { Activation }=\text { relu, weight optimization }=\text { adam, batch size }=512 \text {, number of } \\
\text { epochs }=100 \text {, learning rate }=0.01\end{array}$ \\
\hline
\end{tabular}

\subsection{Performance Comparison with other Deep-Learning-Based Models}

Table 10 presents the performance comparison of deep-learning methods for pressure prediction. $\mathrm{lr}, \mathrm{dt}, \mathrm{rf}, \mathrm{mlp}, \mathrm{cnn}$, and lstm were adopted for pressure prediction, and the results were validated by error metrics: MSE, RMSE, MAE, and MAPE. Therefore, the test results indicate that the proposed CNN-LSTM model outperformed existing deep-learning methods in terms of pressure prediction, and it is thus the most efficient pressure-prediction method.

Table 10. Performance comparison of deep-learning methods.

\begin{tabular}{ccccc}
\hline Method & MSE & RSME & MAE & MAPE \\
\hline CNN & 0.2411 & 0.2835 & 0.2040 & 16.6666 \\
LSTM & 0.2329 & 0.2785 & 0.1970 & 16.1211 \\
The proposed & 0.1246 & 0.2038 & 0.1164 & 11.6133 \\
\hline
\end{tabular}

\section{Conclusions}

Based on the IoT sensor data of the Yongsu WPP currently in operation, this study applied a prediction technique based on ML, an element technology of a digital twin, and demonstrated the superior performance of the proposed method. In the case of the WPP, with the definition of significant data by multiple parameters such as wave height data and sensor data of OWC, correlation analysis was performed with various 
parameters and through the analysis results, a methodology was investigated to predict the required information. Based on the raw dataset, features suitable for the machinelearning model were derived through feature engineering, and using the preprocessed data, training was performed in various models of $\mathrm{ML}$, and a model with a high score was selected. A CNN-LSTM network that models temporal features was proposed to predict the pressure inside the WPP chamber. OWC chamber pressure prediction is challenging because the pressure exhibits an irregular trend due to external variability. This difficulty was resolved by linearly combining CNN and LSTM, and by modeling the complex functions of the actual pressure datasets of the WPP, improved modeling performance was achieved. The performance of the developed prediction model was compared with that of the existing machine learning methods. The performance was evaluated using a 10 -fold cross-validation. The best MSE performance was obtained with the CNN-LSTM. In addition, the results showed that the standard deviation of cross-validation of the CNNLSTM was small compared to the other models. As shown in Figure 12, the CNN-LSTM shows superior performance compared to other deep-learning methods, CNN and LSTM. The OWC chamber pressure prediction requires high performance because it is a pivotal factor influencing the efficiency of the OWC-WEC operation. The proposed method shows high prediction performance after $2.5 \mathrm{~s}$. Through this study, it is expected that the efficiency of operation can be improved by predicting the pressure of OWC with improved accuracy, which is closely related to the power generation output and failure of OWC-WEC, and the utility of machine-learning technology required for smart operation with the digital twin of OWC-WEC was demonstrated.

In this study, because a sufficient amount of data were not available from the IoT sensors, there are some limitations to the full validation of the application of the proposed method. In the future, the design of a predictive analysis model with improved accuracy for smart operation and maintenance of OWC-WEC and real-time large-scale data analysis are required for further research. In particular, because of the problems that arise owing to the increase in the number of input vectors and the consequent sharp increase in the time required for training and analysis, addressing these problems can be achieved by the advancement in the OWC pressure prediction model based on machine learning, research on accuracy improvement using hyperparameter tuning of the deep-learning model, and further research on training environments based on distributed/parallel computing for real-time large-scale sensor data processing.

Author Contributions: Conceptualization, T.H., J.H., D.J.; writing-review and editing, D.S.; writingoriginal draft preparation, T.H.; data analysis, D.S.; data platform implementation, D.S.; project administration, M.K. All authors have read and agreed to the published version of the manuscript.

Funding: This research was funded by both the KRISO (Grant No.: PES3590) and KISTI (Grant No. K-21-L02-C06-S01), South Korea.

Institutional Review Board Statement: Not applicable.

Informed Consent Statement: Not applicable.

Acknowledgments: The authors are grateful to KRISO for providing the opportunity to participate in the project and for providing WEC data.

Conflicts of Interest: All the authors have no conflict of interest to declare.

\section{References}

1. Minister of Trade, Industry and Energy. The 8th Basic Plan for Long-Term Electricity Supply and Demand; Minister of Trade, Industry and Energy: Sejong, Korea, 2017.

2. Could Waves Become the Next Big Renewable Energy Source? Available online: https://edition.cnn.com/2016/12/12/africa/ ghana-wave-energy/ (accessed on 7 December 2020).

3. Cascajo, R.; García, E.; Quiles, E.; Correcher, A.; Morant, F. Integration of marine wave energy converters into seaports: A case study in the Port of Valencia. Energies 2019, 12, 787. [CrossRef] 
4. Kharati-Koopaee, M.; Fathi-Kelestani, A. Assessment of oscillating water column performance: Influence of wave steepness at various chamber lengths and bottom slopes. Renew. Energy 2020, 147, 1595-1608. [CrossRef]

5. Lee, K.H.; Lee, J.H.; Jeong, I.H.; Kim, D.S. 3-Dimensional numerical analysis of air flow inside OWC type WEC equipped with channel of seawater exchange and wave characteristics around its structure (in case of regular waves). JKSCOE 2018, 30, 242-252. [CrossRef]

6. Malmo, O.; Reitan, A. Wave-power absorption by an oscillating water in a channel. J. Fluid Mech. 1985, 158, 153-175. [CrossRef]

7. Heath, T.; Whittaker, T.; Boake, C. The design, construction and operation of the LIMPET wave energy converter (Islay, Scotland) [Land Installed Marine Powered Energy Transformer]. In Proceedings of the 4th European Wave Energy Conference, Aalborg, Denmark, 4 December 2000; pp. 49-55.

8. Boake, C.B.; Whittaker, T.J.; Folley, M.; Ellen, H. Overview and initial operational experience of the LIMPET wave energy plant. In Proceedings of the Twelfth International Offshore and Polar Engineering Conference, Kitakyushu, Japan, 26-31 May 2002; pp. 586-594.

9. Vieira, M.; Monk, K.; Sarmento, A.; Reis, L. The Pico power plant as an infrastructure for development, research and graduation. In Proceedings of Congresso de Ciência e Desenvolvimento dos Açores; ACDA: Angra do Heroísmo, Portugal, 2013; pp. 1-25.

10. Le Crom, I.; Brito-Melo, A.; Neumann, F.; Sarmento, A. Portuguese grid connected OWC power plant: Monitoring Report. In Proceedings of the Twentieth International Offshore and Polar Engineering Conference, Beijing, China, 20-25 June 2010; pp. 20-25.

11. Torre-Enciso, Y.; Ortubia, I.; De Aguileta, L.L.; Marqués, J. Mutriku wave power plant: From the thinking out to the reality. In Proceedings of the 8th European Wave and Tidal Energy Conference, Uppsala, Sweden, 7-10 September 2009; pp. 319-329.

12. Lee, H.; Oh, J.-S. Study of Power Output Characteristics of Wave Energy Conversion System According to Turbine Installation Method Combined with Breakwater. JOET 2015, 29, 317-321. [CrossRef]

13. Kim, J.-S.; Park, J.-Y.; Choi, J.-S.; Shin, S.-H. Analysis of Wave Energy Resource in the West Sea of Jeju based on Wave Observation Data. JKOSMEE 2020, 23, 37-46. [CrossRef]

14. Cui, Y.; Liu, Z.; Zhang, X.; Xu, C. Review of CFD studies on axial-flow self-rectifying turbines for OWC wave energy conversion. Ocean Eng. 2019, 175, 80-102. [CrossRef]

15. Roh, C.; Kim, K.; Kim, J.; Hong, K. A Comparison of PWM methods for Power Converter of OWC Wave Power Generation System. JKOSMEE 2019, 22, 84-94. [CrossRef]

16. Park, S.; Kim, K.-H.; Nam, B.; Kim, J.-S.; Hong, K. A Study on the Performance Evaluation of the OWC WEC Applicable to Breakwaters using CFD. JKSMEE 2018, 21, 317-327. [CrossRef]

17. Fillbrunn, A.; Dietz, C.; Pfeuffer, J.; Rahn, R.; Landrum, G.A.; Berthold, M.R. KNIME for reproducible cross-domain analysis of life science data. J. Biotechnol. 2017, 261, 149-156. [CrossRef] [PubMed]

18. Abdelhafez, H.A.; Amer, A.A. The Challenges of Big Data Visual Analytics and Recent Platforms. WSCIT 2019, 9, 28-33.

19. Bonthu, S.; Bindu, K.H. Review of Leading Data Analytics Tools. IJET 2017, 7, 10-15. [CrossRef]

20. Kim, Y.-G.; Moon, K.-I. Clustering of Smart Meter Big Data Based on KNIME Analytic Platform. JIIBC 2020, 20, 13-20. [CrossRef]

21. Datasolution. Predictive Analysis Solution, KNIME Analytics Platform. Available online: http://www.datasolution.kr (accessed on 4 December 2020).

22. Bartlett, P.L.; Long, P.M.; Lugosi, G.; Tsigler, A. Benign overfitting in linear regression. PNAS 2020, 117, 30063-30070 [CrossRef] [PubMed]

23. Sevkli, M.; Sevkli, A.Z.; Cosgun, O. Comparison of Data Mining and Mathematical Models for Estimating Fuel Consumption of Passenger Vehicles. In Proceedings of the MWAIS 2019, Oshkosh, WI, USA, 21-22 May 2019; pp. 1-6.

24. Qin, D.; Yu, J.; Zou, G.; Yong, R.; Zhao, Q.; Zhang, B. A novel combined prediction scheme based on CNN and LSTM for urban PM 2.5 concentration. IEEE Access 2019, 7, 20050-20059. [CrossRef]

25. Fusco, F.; Ringwood, J.V. Short-term wave forecasting for real-time control of wave energy converters. IEEE Trans. Sustain. Energy 2010, 1, 99-106. [CrossRef]

26. Simonetti, I. Optimization of Oscillating Water Column Wave Energy Converters: A Numerical Study. Ph.D. Thesis, Technische Universität Braunschweig, Florence, Italy, 2017.

27. Sheng, W.; Lewis, T. Artificial neural network application in short-term prediction in an oscillating water column. In Proceedings of the Twentieth International Offshore and Polar Engineering Conference, Beijing, China, 20-25 June 2010.

28. Paparella, F.; Monk, K.; Winands, V.; Lopes, M.; Conley, D.; Ringwood, J.V. Up-wave and autoregressive methods for short-term wave forecasting for an oscillating water column. IEEE Trans. Sustain. Energy 2014, 6, 171-178. [CrossRef]

29. Monk, K.; Conley, D.; Winands, V.; Lopes, M.; Zou, Q.; Greaves, D. Simulations and field tests of pneumatic power regulation by valve control using short-term forecasting at the Pico OWC. In Proceedings of the 11th European Wave Tidal Energy Conference, Nantes, French, 6-11 September 2015; pp. 1-10.

30. Ni, C.; Ma, X. Prediction of wave power generation using a convolutional neural network with multiple inputs. Energies 2018, 11, 2097. [CrossRef]

31. Choi, E.; Cho, S.; Kim, D.K. Power Demand Forecasting Using Long Short-Term Memory (LSTM) Deep-Learning Model for Monitoring Energy Sustainability. Sustainability 2020, 12, 1109. [CrossRef] 